\title{
The effects of coordinative locomotor training on coordination and gait in chronic stroke patients: a randomized controlled pilot trial
}

\author{
Jin-Cheol Kim*, Jae-Heon Lim \\ The Lab Cooperative, The Lab, Gwangju, Korea
}

The purpose of this study was to investigate the effects of coordinative locomotor training (CLT) on coordination and gait in chronic stroke patients. Thirteen stroke patients were randomly assigned to one of two groups: The experimental group ( $E G, n=7$ ) and control group (CG, $n=6)$. After balanced random assignment, interventions were provided to all patients in twelve 30-min sessions during a 4-week period (3 sessions a week). The EG group underwent CLT after performing the sprinter and skater patterns in four postures. The CG group was provided with conventional neurodevelopment treatment. The primary outcomes were measured using the gait assessment and intervention tool (GAIT) as well as G-WALK. The secondary outcomes were measured using a 10 -m walk test (10 MWT) and Timed Up and Go Test. There was no statistically significant difference in the mean values between the two groups. Within both groups there were significant differences in GAIT and 10MWT. There were significant differences in gait speed and stride length in the experimental group, but not in the control group. CLT showed a more positive improvement in gait and coordination after stroke than conventional rehabilitation exercise.

Keywords: Coordination, Coordinative locomotor training, Gait, Stroke

\section{INTRODUCTION}

Human locomotion is responsible for the movement and coordination of the limbs while the head and body remain stable (Pozzo et al., 1990). Locomotion is a complex process occurring continuously through space and time. Coordination the synergy and combination of various muscles and joints are prominent in gait activities (Dietz et al., 2001). Locomotion requires harmonious connections of the body's joints and muscles to successfully perform simple daily movements to complex movements. When a person tries to carry out a purposeful movement, the elements that cause the movement form several synergies, combine with one another, and appear in harmony with all the limbs, and this is what is referred to as "coordination" (Turvey, 1990). Coordination is an essential factor in the process of minimizing and adjusting the degrees of freedom of movement to achieve locomotion-i.e., gait activity.

Human gait activity occurs with close interactions between the arms and the legs. The most common and natural movement is when the right arm/left leg and the left arm/right leg are simultaneously swinging alternately (Newell and Molenaar, 1998). The nervous system controls the circuit so that the upper and lower limbs can alternately swing unconsciously during walking. The synergy of the muscle groups under control is gathered in one unit to show the most effective muscle activation pattern (Clark et al., 2010). Several body segments, which are grouped into one unit, are structured into individual systems, called "coordinative structure" (Kelso and Schöner, 1988). There are two types of coordination: intralimb coordination, which appears for one leg and one arm to move effectively, and interlimb coordination, which appears for two or more limbs (Aoi and Funato, 2016). There is coordinative movement of the intralimb and interlimb segments so that his or her body could move forward efficiently while he or she is walking upright with two feet.

${ }^{*}$ Corresponding author: Jin-Cheol Kim (D) https://orcid.org/0000-0003-4375-9239 The Lab Cooperative, The Lab, 121 Yangnim-ro, Dong-gu, Gwanju 61489, Korea Tel: +82-62-460-7574, Fax: +82-62-460-7633, E-mail: kjcbboy@gmail.com Received: August 6, 2018 / Accepted: November 12, 2018
This is an Open Access article distributed under the terms of the Creative Commons Attribution Non-Commercial License (http://creativecommons.org/licenses/by-nc/4.0/) which permits unrestricted non-commercial use, distribution, and reproduction in any medium, provided the original work is properly cited. 
However, stroke patients still have gait coordination deficiencies, despite receiving various rehabilitation treatments and coordination-related intervention training (Biernaskie and Corbett, 2001). The spatiotemporal coordination among the head, trunk, and pelvis changes, or the pelvis, knees, and ankles cannot be controlled (Lamontagne et al., 2005). Asymmetric movements of the upper and lower limbs during stance and swing phases show large energy consumption and an inefficient gait pattern (Bütefisch et al., 1995). This is due to reduced propulsion, stride length, and asymmetric weight support, as well as lowered muscular endurance, which appear between the paralyzed and affected sides (Bovonsunthonchai et al., 2012), as the intralimb and interlimb coordination is not done properly (Daly et al., 2006). The reduced gait ability of stroke patients results in spatiotemporally asymmetrical gait patterns, such as decreases in gait speed, cadence, step length, and stride length, compared with nonstroke adults (Duncan et al., 2005).

To improve the gait ability in a rehabilitation environment, visual feedback training, underwater gait training, treadmill training, virtual reality, and task-oriented training are offered. However, these training methods are focused on improving the quantitative aspect of gait. For efficient gait training, quality training is important to achieve gait symmetry by pushing the body forward through rhythmic movements and alternating motions of both legs (Mauritz, 2002).

Ways to improve asymmetric problems associated with gait has recently been studied in various fields, such as biomechanics, neuroscience, and motor control, which are related to intralimb and interlimb coordination (Reisman et al., 2005). Based on these studies, coordinative locomotor training (CLT), in which the coordination between the limbs is pattern has been provided in the clinical field for exercise purposes. CLT is a functional exercise designed to move the limbs alternatively, with the head and trunk acting as the center of movement. This training is done by using sprinter and skater patterns that mimics the motion of sprinters and skaters. CLT is a training method for improving the intralimb and interlimb coordination and enhancing the gait by pattern movements appearing in the gait into a three-dimensional movement form. The studies on the effects of CLT on stroke patients with reduced gait activity due to decreased coordination abilities are insufficient. The purpose of this study was to investigate the effects of CLT on gait and coordination in chronic stroke patients, and to confirm the possibility of using CLT in the future as an intervention tool to improve gait activity.

\section{MATERIALS AND METHODS}

\section{Study subjects and period}

This study included 13 stroke patients admitted to the rehabilitation department in a long-term care hospital in Gwangju, Korea. The study requirements were explained and written informed consent was obtained from all participants prior to the study. Subjects were selected based on the inclusion and exclusion criteria. The inclusion criteria were as follows: (a) those who have been diagnosed with stroke at the hospital for more than 6 months; (b) those who have no cognitive impairment (Korean version of Mini-Mental State Examination [MMSE-K] score of 24 or more); (c) those who have no abnormality in visual and auditory senses and proprioception; (d) those with Fair+ or higher on manual muscle tests of the upper and lower limbs in the affected side; and (e) those who can use assistive devices or walk independently. The exclusion criteria, on the other hand were as follows: (a) those with orthopedic disorders in the lower limbs; (b) those with other diseases that may affect gait; and (c) those who were participating in a rehabilitation program other than the ones provided by this study. This study was approved by the Ethics Committee of Honam University (approval number, 1041223-201607-HR-018).

\section{Study design and process}

This study had a pre-post design, and randomly assigned 13 subjects to the experimental and control groups. For the randomization process, sealed envelopes_ —each containing a piece of paper marked $\mathrm{O}$ or $\mathrm{X}$-were prepared, and the process was carried out by a physical therapist who did not participate in the subject's intervention or evaluation prior to the pretest.

The 13 study participants underwent a pretest. The participants in the two groups performed the exercise patterns in the postures, and gait training was performed for a total of 12, 30min sessions that were spread out in a 4-week period (3 sessions per week). After the 4-week intervention, participants in the two groups underwent the posttest (Fig. 1).

\section{Training methods}

\section{CLT for the experimental group}

During the intervention period, the experimental group was provided with a CLT program consisting of four basic postures (supine, bridge, sitting, and standing), along with gait training. The total training time was $30 \mathrm{~min}$ per day: $20 \mathrm{~min}$ for the four postures ( $5 \mathrm{~min}$ each) and $10 \mathrm{~min}$ for gait training. The sprinter and skater patterns were performed for $2 \mathrm{~min}$ and $30 \mathrm{sec}$ for each 


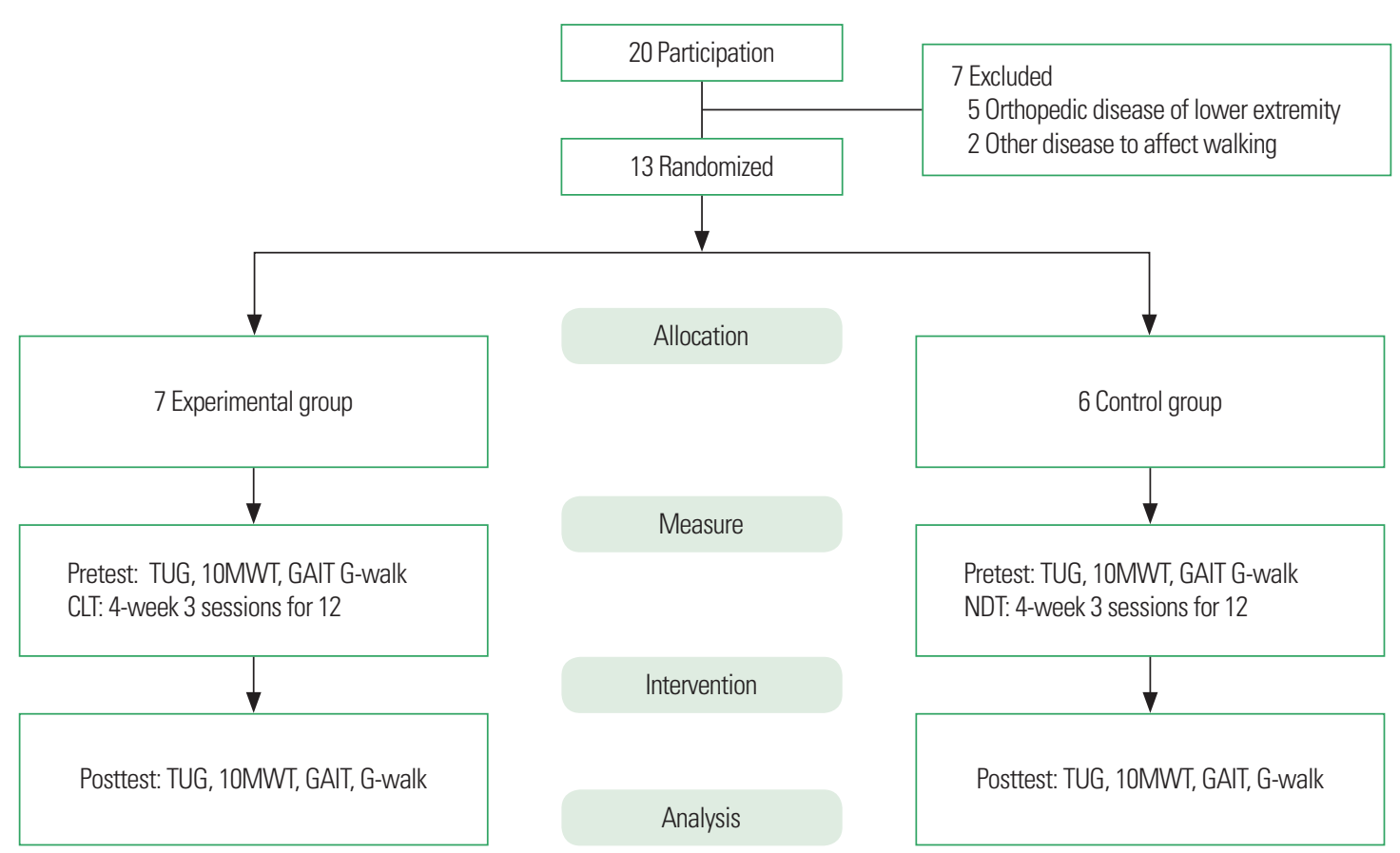

Fig. 1. The study flowchart. TUG, Timed Up and Go Test; 10MWT, 10-min walk test; GAIT, gait assessment and intervention tool; CLT, coordinative locomotor training; NDT, neuro-developmental treatment.

pattern in supine, bridge, sitting, and standing postures. The sprinter pattern was performed by imitating a runner's movement, including the flexion-adduction-external rotation pattern in the paralyzed upper limb and the extension-abduction-internal rotation pattern in the lower limb of the same side. For the opposite upper limb, the extension-abduction-internal rotation pattern was applied, and for the lower limb of the same side, the flexion-adduction-external rotation pattern was applied. The skater pattern was performed by imitating the skater's actions, including the flexion-abduction-external rotation pattern in the paralyzed upper limb and the extension-adduction-external rotation pattern in the lower limb of the same side. For the upper limb on the opposite side, the extension-adduction-internal rotation pattern was applied, while the flexion-abduction-internal rotation pattern was applied on the lower limb on the same side. If the patient was unable to raise the paralyzed upper or lower limb to execute the pattern properly in each posture, therapist's assistance was provided.

\section{Neuro-developmental treatment for the control group}

During the intervention period, a physical therapist with clinical experience of 2 or more years performed the 30-min treatment for central-nervous-system development in the control group. The intervention was conducted with focus on the mat and gait training. Patients practiced the transitional posture to be able to change from the lying to standing posture, with therapist's assistance if necessary. After exercising the shifting of the weight to the front, back, left, and right in standing posture, gait training was carried out by removing one foot at a time with assistance.

\section{Evaluation instruments \\ Gait assessment and intervention tool (GAIT)}

To examine the intralimb and interlimb coordination during walking, gait assessment and intervention tool (GAIT) was used. This measurement tool is an observational scoring-based scale consisting of 31 items in three categories to measure the cooperative movement a component of gait. The components of GAIT are given a total of 62 points: 4 items for "A: Stance and Swing Phases," 14 items for "B: Stance Phase," and 13 items for "C: Swing Phase." The intrarater reliability of GAIT (intraclass correlation coefficient, ICC) was 0.98 ( $P=0.0001$, confidence interval $[C I]=95 \%)$ and the interrater reliability (ICC) was 0.83 ( $P=0.007, \mathrm{CI}=95 \%$ ) (Daly et al., 2009).

\section{0-m walk test}

To measure gait speed during walking, a 10-m walk test (10MWT), which is a functional assessment tool commonly used in clinical practice, was performed. A 14-m-section straight-line distance between the 2 points was marked, and patients were 
guided to walk at a comfortable speed. Considering the acceleration and deceleration, the first $2 \mathrm{~m}$ and the last $2 \mathrm{~m}$ of the walk were excluded from measurement, and the gait time for the $10-\mathrm{m}$ distance was measured 3 times to obtain the average. The intraand interrater reliabilities of the 10MWT were $r=0.89-1.00$ (Fulk and Echternach, 2008).

\section{Timed Up and Go Test}

The Timed Up and Go Test (TUG) was measured to determine the functional movement and locomotion necessary for sitting, standing, and walking. Participants sat in a chair with an armrest and were instructed to stand upon the verbal instruction "Start," pass the turnaround at the 3-m distance, and sit back on the chair. The measurements were repeated 3 times using a stopwatch, and the average value was recorded. The intra- and interrater reliability scores of TUG were $r=0.99$ and $r=0.98$, respectively (Botolfsen et al., 2008).

\section{G-WALK}

To measure the movement of the pelvis and the spatiotemporal parameters during walking, G-WALK AP1177 (BTS G-WALK, BTS Bioengineering Corp., Quincy, MA, USA) was used. G-WALK is a wireless system consisting of 3 -axis accelerometer, magnetic sensor, and 3-axis gyroscope. The 3-axis accelerometer is equipped with a Bluetooth sensor. A laptop with a BTS G-studio software program capable of handling this signal transmits the signal to the G-sensor with the Bluetooth device. The 3-axis accelerometer is worn on the surface of the body near the fifth lumbar vertebrae using a strap belt. The measurer ran the BTS G-studio software program linked to G-WALK with the verbal instruction, "Walk in the fixed section at a comfortable speed" to the subject. The measurement started from the moment the first foot landed on the ground, and the signal was collected the moment the last foot passed over the finish line. Subjects were asked to walk $10 \mathrm{~m}$ of the fixed section for a total of 3 times, and the parameters were measured repeatedly. The gait parameter data collected during walking were analyzed by the BTS G-Studio software program. In terms of reliability, the intraclass correlation coefficient was $r=$ 0.98 in G-WALK and $r=0.99$ in cadence (Awotidebe et al., 2016).

\section{Statistical analysis}

All the statistical analyses were performed using IBM SPSS Statistics ver. 21.0 (IBM Co., Armonk, NY, USA). The general characteristics of the subjects were expressed as the mean and standard deviation through descriptive statistics. The Kolmogorov-Smirn- ov test was used to test the normality. The results showed that all variables did not satisfy the normal distribution. Thus, MannWhitney $U$-test (a nonparametric test) was used to examine the homogeneity of the general and clinical characteristics of the subjects in each group. The Mann-Whitney $U$-test was conducted to determine the mean difference before and after the training between the two groups. The Wilcoxon signed-rank test was performed to determine the difference before and after the training within each group. Using Cohen effect size for the time change within the group, large effect of 0.50 , moderate effect of 0.30 , and small effect of 0.10 were suggested. The $95 \%$ confidence interval was presented when the time change was significant. For all tests, the statistical significance was set at 0.05 .

\section{RESULTS}

\section{General characteristics of the study participants}

A total of 13 subjects were included in this study, 7 in the experimental group and 6 in the control group. There were no significant differences in gender, stroke type, paralysis side, age, date of onset, MMSE-K, 10MWT, GAIT, and TUG between the two groups. The general and clinical characteristics of the subjects are shown in Table 1.

\section{Primary and secondary outcomes}

Table 2 compares the results of the primary and secondary outcomes before and after training, as well as within and between the experimental and control groups. There was no significant difference in the mean between the experimental and control groups, but there was a significant difference in GAIT, indicating coordination according to the time change in both the experimental and

Table 1. General characteristics of the subjects $(n=13)$

\begin{tabular}{lccc} 
Characteristic & $\begin{array}{c}\text { Experimental group } \\
(\mathrm{n}=7)\end{array}$ & $\begin{array}{c}\text { Control group } \\
(\mathrm{n}=6)\end{array}$ & Z-values \\
\hline Sex, male:female & $6: 1$ & $5: 1$ & \\
Ischemic:hemorage stroke & $4: 3$ & $4: 2$ & \\
Plegic side, right:left & $3: 4$ & $3: 3$ & \\
Age (yr) & $59.57 \pm 11.75$ & $64.50 \pm 13.03$ & 0.62 \\
Days after stroke & $33.85 \pm 29.61$ & $29.16 \pm 34.86$ & 0.53 \\
MMSE-K & $25.71 \pm 1.11$ & $26.50 \pm 1.04$ & 0.29 \\
1OMWT & $22.21 \pm 18.11$ & $25.65 \pm 15.05$ & 0.23 \\
TUG & $24.94 \pm 19.32$ & $28.09 \pm 15.54$ & 0.73 \\
GAIT & $24.71 \pm 11.20$ & $27.50 \pm 14.11$ & 1.00 \\
\hline
\end{tabular}

Values are presented as number or mean \pm standard deviation. MMSE-K, Korean version of Mini-Mental State Examination; 10MWT, 10-m walk test; TUG, Timed Up and Go Test; GAIT, gait assessment intervention tool. 
Table 2. The within-group and between-group comparisons for the outcome measures

\begin{tabular}{|c|c|c|c|c|c|c|c|c|c|c|c|}
\hline \multirow{2}{*}{ Variable } & \multicolumn{2}{|c|}{ Experimental group $(n=7)$} & \multirow{2}{*}{$\begin{array}{l}\text { Difference } \\
\text { (post-pre) }\end{array}$} & \multirow{2}{*}{ Z-value } & \multirow{2}{*}{$\begin{array}{c}\text { Effect } \\
\text { size }\end{array}$} & \multicolumn{2}{|c|}{ Control group $(n=6)$} & \multirow{2}{*}{$\begin{array}{l}\text { Difference } \\
\text { (post-pre) }\end{array}$} & \multirow{2}{*}{ Z-value } & \multirow{2}{*}{ Z-value ${ }^{\text {bl }}$} & \multirow{2}{*}{$\begin{array}{r}\text { Effect } \\
\text { size }\end{array}$} \\
\hline & Pretest & Posttest & & & & Pretest & Posttest & & & & \\
\hline \multicolumn{12}{|c|}{ Primary outcome measure } \\
\hline GAIT & $24.71 \pm 11.20$ & $18.28 \pm 8.88$ & $-6.42 \pm 4.99$ & $-2.37^{*}$ & 0.64 & $27.50 \pm 14.11$ & $23.00 \pm 12.85$ & $-4.50 \pm 6.74$ & $-2.03^{*}$ & 0.53 & 0.33 \\
\hline \multicolumn{12}{|c|}{ Temporal gait parameters } \\
\hline Gait speed $(\mathrm{cm} / \mathrm{sec})$ & $6.27 \pm 1.40$ & $8.74 \pm 3.88$ & $0.25 \pm 0.30$ & $-1.99 *$ & 0.85 & $7.30 \pm 2.52$ & $7.23 \pm 2.43$ & $-0.01 \pm 0.10$ & -0.31 & 0.13 & 0.03 \\
\hline Cadence (steps/min) & $94.58 \pm 30.01$ & $97.67 \pm 34.19$ & $3.09 \pm 5.37$ & -1.52 & 0.09 & $83.93 \pm 29.22$ & $80.86 \pm 29.48$ & $-3.07 \pm 7.01$ & -0.93 & 0.05 & 0.10 \\
\hline $\operatorname{SLSP}(\%)$ & $31.24 \pm 6.99$ & $33.78 \pm 7.22$ & $2.54 \pm 3.19$ & -1.52 & 0.36 & $40.03 \pm 9.58$ & $40.33 \pm 10.31$ & $0.30 \pm 1.17$ & -0.52 & 0.36 & 0.03 \\
\hline $\operatorname{DLSP}(\%)$ & $12.17 \pm 3.53$ & $12.33 \pm 5.12$ & $0.16 \pm 2.45$ & -0.16 & 0.04 & $10.08 \pm 4.42$ & $11.61 \pm 5.50$ & $1.53 \pm 2.84$ & -1.36 & 0.29 & 0.31 \\
\hline \multicolumn{12}{|l|}{ Spatial gait parameters } \\
\hline Step length (\%) & $49.59 \pm 2.35$ & $49.70 \pm 2.71$ & $0.11 \pm 1.18$ & -0.25 & 0.04 & $48.76 \pm 2.66$ & $50.69 \pm 3.84$ & $1.93 \pm 5.77$ & -0.63 & 0.94 & 0.58 \\
\hline Stride length (cm) & $85.85 \pm 26.21$ & $113.85 \pm 30.08$ & $28.00 \pm 29.38$ & $-2.19^{*}$ & 0.99 & $87.33 \pm 39.89$ & $111.50 \pm 18.72$ & $24.16 \pm 42.48$ & -0.10 & 0.73 & 0.77 \\
\hline \multicolumn{12}{|c|}{ Secondary outcome measure } \\
\hline 10MWT & $22.20 \pm 18.11$ & $18.56 \pm 14.97$ & $-3.64 \pm 3.45$ & $-2.36^{*}$ & 0.22 & $25.65 \pm 15.04$ & $22.56 \pm 11.98$ & $-3.08 \pm 3.56$ & $-2.20^{*}$ & 0.83 & 0.23 \\
\hline TUG & $24.94 \pm 19.32$ & $23.59 \pm 19.41$ & $-1.35 \pm 3.70$ & -1.18 & 0.07 & $28.09 \pm 15.54$ & $24.52 \pm 11.08$ & $-3.56 \pm 5.10$ & -1.57 & 0.73 & 0.26 \\
\hline
\end{tabular}

Values are presented as mean \pm standard deviation.

GAIT, gait assessment intervention tool; SLSP, single limb support period; DLSP, double limb support period; 10MWT, 10-m walk test; TUG, Timed Up and Go Test.

a) Significant difference within-group pre and posttest. ${ }^{b /}$ Significant difference between-group comparison. ${ }^{*} P<0.05$.

control groups (EG: $Z,-2,37 ; P<0.02 ; 95 \%$ CI, 1.80-11.04; CG: $Z,-2.03 ; P<0.04 ; 95 \% \mathrm{CI},-2.57$ to 11.57$)$. For gait speed and temporal parameter of gait, there was a significant difference after the training compared with before in the experimental group (EG: $Z,-1.99 ; P<0.04 ; 95 \%$ CI, -5.28 to 0.33 ; CG: $Z, 0.31 ; P<0.75$; $95 \% \mathrm{CI} ;-1.01$ to 1.15$)$. There was also a significant difference in 10MWT in both the experimental and control groups (EG: $Z$, -2.36; $P<0.02$; $95 \%$ CI, -5.91 to -1.48 ; CG: $Z,-2.20 ; P<0.03$; $95 \% \mathrm{CI},-6.12$ to -1.29$)$. For stride length and spatial parameter of gait, there was a significant difference after the training compared with before the training in the experimental group (EG: $Z$, -2.19; $P<0.028$; 95\% CI, -6.52 to -0.19 ; CG: $Z,-0.1 ; P>0.92$; $95 \% \mathrm{CI},-7.91$ to 10.22$)$. Other than these, there was no significant difference within the group according to the time change in cadence, SLSP, DLSP, step length, and TUG.

\section{DISCUSSION}

Human movement requires flexibility to be able to respond appropriately to the environment when performing specific tasks (Willis et al., 2004). However, in the case of motor control-related injuries, problems in achieving harmonious exercise techniques may arise due to reduced balance, decreased coordination, and limited gait activity (Shumway-Cook and Woollacott, 2000). In particular, patients with stroke exhibit asymmetric movement patterns accompanied by weakened muscle strength, changes in muscle tension, and decreased coordination, which are manifested as loss of motor function. This study was conducted to investigate the effects of CLT on gait and coordination in stroke patients.

The results of this study showed no significant difference between the experimental and control groups. However, the former group showed significant differences in GAIT, 10MWT, gait speed, and stride length after than before the intervention at each time point. Balance-the process by which continuous stability is maintained - is the most essential factor in a human being's daily life activities or in carrying out purposeful movements (Cohen et al., 1993). The ability to maintain and control a stable posture from sudden fluctuations that may arise in everyday situations is closely related to effective motor skills (Carr et al., 1985). In this study there was no significant difference between the two groups in the TUG measurement that was performed to determine the dynamic balance ability required for walking. This suggests that the physical functions related to the coordination and cognitive abilities of the vestibular, visual, sensory, and musculoskeletal systems involved in balancing the body did not transfer to the functional activities related to walking. The reason for this is that the intervention used in this study had the subjects repeatedly practice static motions in various postures, instead of having them repeatedly practice the motions in dynamic postures. It is also supported by the fact that each posture and situation require different skills.

Coordination between various body parts while moving is a prerequisite for performing functional gait. Coordination provides information by organizing the various and free degrees of freedom 
in the central nervous system for carrying out functional activities. Abundant degrees of freedom mobilize the nervous system to execute more exercise patterns and solve complex problems. In the present study, GAIT was used to measure and compare the coordination. There was significant improvement after the intervention compared with before the training in both groups. The study by Daly et al. (2011), which evaluated 53 stroke patients with coordination problems, reported significant differences in the coordination abilities required for gait in both the experimental and control groups, as a result of applying muscle strength, coordination exercise, and ground walking training. This result is consistent with those of the previous studies. The restoration of coordinative movement may have affected the amount and magnitude of movement of the body by improving the movement control function of the central nervous system, thereby increasing the movement, torque, and angular velocity of the joints.

Normal gait, where numerous joints and muscles function as a coordinative structure and result in complex and integrated activities, consumes less energy, resulting in greater efficiency. Therefore, gait requires contraction of the normal antigravity muscle and mutually alternate and harmonious movement of the upper and lower limbs (Blennerhassett and Dite, 2004). For continuous gait, flexibility is needed for the legs to support the body, propel to the intended direction, produce basic movement rhythms, have dynamic balance capability, and properly cope with and adapt to the changing environmental needs (Coutts, 1999). In this study, gait speed was measured using 10MWT, and both groups showed significant improvement after the training. Stephenson et al. (2009) reported significant improvement in gait speed and coordination of the arms in both the experimental group (10 stroke patients) and control group (10 healthy subjects) from treadmill training. Considering the previous studies the gait training of CLT and the gait training applied to the control group in the present study may have improved the gait speed of each subject.

The gait cycle is roughly divided into two phases: the stance and swing phases. The stance phase is when the foot is touching the ground, while the swing phase is when the foot is in the air. Assuming that the total gait cycle is $100 \%$, both phases account for $60 \%$ and $40 \%$, respectively. When each phase is subdivided, the stance phase is divided into two tasks: the weight-acceptance task and single-limb support task, involving standing on one foot. The swing phase can be subdivided into the limb advancement task lifting a foot from the ground (Whittle, 1996). In the present study G-WALK was used to examine the spatiotemporal variables according to gait cycle. The study results showed a significant dif- ference in gait speed, a temporal variable of gait, after the training compared with before the training in the experimental group, but not in the control group. Moreover, there was a significant difference in stride length among the spatial variables after the training compared with before the training. In the study by Plummer et al. (2007), a task-oriented training program related to locomotor movement was applied to stroke patients, and significant improvements were shown in the gait speed and stride length. This result is consistent with those of previous studies; the stride length refers to the distance from the heel of one foot touching the ground to the heel of the same foot touching the ground again. In the present study, the step length was increased through CLT in the experimental group, and it was thought that the longer step led to gait speed improvement.

Due to the small sample size of this study, it may be difficult to generalize the study results. The effects of CLT were assessed through a 4-week training period, making it impossible to determine the long-term effects of CLT. Moreover, it is impossible to know the persistence of the effect because follow-up evaluation was not done. Future studies should be conducted with a greater sample size and with a follow-up evaluation to investigate the persistence of the effects of CLT.

The results of this study showed that the CLT can improve gait quality of chronic stroke patients. It can be used as a self-exercise program or as a postdischarge home exercise program by applying patterns in various postures. It can also be useful in constructing a program for solving gait and coordination problems in various fields.

\section{CONFLICT OF INTEREST}

No potential conflict of interest relevant to this article was reported.

\section{REFERENCES}

Aoi S, Funato T. Neuromusculoskeletal models based on the muscle synergy hypothesis for the investigation of adaptive motor control in locomotion via sensory-motor coordination. Neurosci Res 2016;104:88-95.

Awotidebe TO, Ativie RN, Oke KI, Akindele MO, Adedoyin RA, Olaogun $\mathrm{MO}$, Olubayode TE, Kolawole BA. Relationships among exercise capacity, dynamic balance and gait characteristics of Nigerian patients with type-2 diabetes: an indication for fall prevention. J Exerc Rehabil 2016;12:581-588.

Biernaskie J, Corbett D. Enriched rehabilitative training promotes im- 
proved forelimb motor function and enhanced dendritic growth after focal ischemic injury. J Neurosci 2001;21:5272-5280.

Blennerhassett J, Dite W. Additional task-related practice improves mobility and upper limb function early after stroke: a randomised controlled trial. Aust J Physiother 2004;50:219-224.

Botolfsen P, Helbostad JL, Moe-Nilssen R, Wall JC. Reliability and concurrent validity of the Expanded Timed Up-and-Go test in older people with impaired mobility. Physiother Res Int 2008;13:94-106.

Bovonsunthonchai S, Hiengkaew V, Vachalathiti R, Vongsirinavarat M, Tretriluxana J. Effect of speed on the upper and contralateral lower limb coordination during gait in individuals with stroke. Kaohsiung J Med Sci 2012;28:667-672.

Bütefisch C, Hummelsheim H, Denzler P, Mauritz KH. Repetitive training of isolated movements improves the outcome of motor rehabilitation of the centrally paretic hand. J Neurol Sci 1995;130:59-68.

Carr JH, Shepherd RB, Nordholm L, Lynne D. Investigation of a new motor assessment scale for stroke patients. Phys Ther 1985;65:175-180.

Clark DJ, Ting LH, Zajac FE, Neptune RR, Kautz SA. Merging of healthy motor modules predicts reduced locomotor performance and muscle coordination complexity post-stroke. J Neurophysiol 2010;103:844-857.

Cohen H, Blatchly CA, Gombash LL. A study of the clinical test of sensory interaction and balance. Phys Ther 1993;73:346-351.

Coutts F. Gait analysis in the therapeutic environment. Man Ther 1999;4: 2-10.

Daly JJ, Nethery J, McCabe JP, Brenner I, Rogers J, Gansen J, Butler K, Burdsall R, Roenigk K, Holcomb J. Development and testing of the Gait Assessment and Intervention Tool (G.A.I.T.): a measure of coordinated gait components. J Neurosci Methods 2009;178:334-339.

Daly JJ, Roenigk K, Holcomb J, Rogers JM, Butler K, Gansen J, McCabe J, Fredrickson E, Marsolais EB, Ruff RL. A randomized controlled trial of functional neuromuscular stimulation in chronic stroke subjects. Stroke 2006;37:172-178.

Daly JJ, Zimbelman J, Roenigk KL, McCabe JP, Rogers JM, Butler K, Burdsall R, Holcomb JP, Marsolais EB, Ruff RL. Recovery of coordinated gait: randomized controlled stroke trial of functional electrical stimulation (FES) versus no FES, with weight-supported treadmill and over-ground training. Neurorehabil Neural Repair 2011;25:588596.

Dietz V, Fouad K, Bastiaanse CM. Neuronal coordination of arm and leg movements during human locomotion. Eur J Neurosci 2001;14:1906-
1914.

Duncan PW, Zorowitz R, Bates B, Choi JY, Glasberg JJ, Graham GD, Katz RC, Lamberty K, Reker D. Management of adult stroke rehabilitation care: a clinical practice guideline. Stroke 2005;36:e100-143.

Fulk GD, Echternach JL. Test-retest reliability and minimal detectable change of gait speed in individuals undergoing rehabilitation after stroke. J Neurol Phys Ther 2008;32:8-13.

Kelso JA, Schöner G. Self-organization of coordinative movement patterns. Hum Mov Sci 1988;7:27-46.

Lamontagne A, De Serres SJ, Fung J, Paquet N. Stroke affects the coordination and stabilization of head, thorax and pelvis during voluntary horizontal head motions performed in walking. Clin Neurophysiol 2005;116:101-111.

Mauritz KH. Gait training in hemiplegia. Eur J Neurol 2002;9 Suppl 1: 23-29.

Newell KM, Molenaar PC. Applications of nonlinear dynamics to developmental process modeling. Mahwah: Lawrence Erlbaum; 1998.

Plummer P, Behrman AL, Duncan PW, Spigel P, Saracino D, Martin J, Fox E, Thigpen M, Kautz SA. Effects of stroke severity and training duration on locomotor recovery after stroke: a pilot study. Neurorehabil Neural Repair 2007;21:137-151.

Pozzo T, Berthoz A, Lefort L. Head stabilization during various locomotor tasks in humans. I. Normal subjects. Exp Brain Res 1990;82:97-106.

Reisman DS, Block HJ, Bastian AJ. Interlimb coordination during locomotion: what can be adapted and stored? J Neurophysiol 2005;94:24032415.

Shumway-Cook A, Woollacott M. Attentional demands and postural control: the effect of sensory context. J Gerontol A Biol Sci Med Sci 2000; 55:M10-16.

Stephenson JL, Lamontagne A, De Serres SJ. The coordination of upper and lower limb movements during gait in healthy and stroke individuals. Gait Posture 2009;29:11-16.

Turvey MT. Coordination. Am Psychol 1990;45:938-953.

Whittle MW. Clinical gait analysis: a review. Hum Mov Sci 1996;15:369387.

Willis A, Gjersoe N, Havard C, Kerridge J, Kukla R. Human movement behaviour in urban spaces: implications for the design and modelling of effective pedestrian environments. Environ Plan B: Urban Anal City Sci 2004;31:805-828. 